文章编号：1001-3555(2020)05-0484-11

\title{
复合金属氧化物催化剂用于甘油氧化制丙烯酸的研究进展
}

\author{
卢自轩 ${ }^{1}$, 李双明 ${ }^{1,2}$, 于三三 ${ }^{1,2}$ \\ (1. 沈阳化厂大学化学丁程兴院, 辽宁 沈阳 110142; \\ 2. 辽宁少化工分离技术重点实验室，辽宁沈阳 110142)
}

\begin{abstract}
摘要: 随着化石燃料的短缺及其利用所产生的环境问题, 可再生生物质资源逐渐成为生产燃料以及化学品的重要 来源. 近年来, 甘油作为生物柴油生产的主要副产物受到了人们的广泛关注，利用其生产高附加值产品以及开发 相关的转化技术也成为了国内外学者研究的热点. 在诸多转化技术中, 甘油选择性氧化制丙烯酸展现出了广阔的 发展前景, 对该反应尤其是催化剂开展研究具有重要的经济意义和社会意义. 我们综述了用于甘油选择性氧化制 丙烯酸复合金属氧化物催化剂的研究现状, 介绍了当前用于该反应的催化体系类型以及对甘油氧化制丙烯酸催化 反应机理的认识, 分析并提出了存在的问题以及对未来的展望.
\end{abstract}

关键词: 甘油; 丙烯酸; 氧化反应; 催化剂; 反应机理

中图分类号: TQ032.4; 0643.36

文献标志码: A

随着可再生资源的兴起, 对其进行高效的转化 利用越来越受到人们的广泛关注 ${ }^{[1-7]}$. 目前, 人们 将大量精力投人到将可再生原料转化为精细化学品 和清洁燃料的绿色催化研究中 ${ }^{8-10]}$. 近十年来, 生 物柴油作为代表性可再生液体燃料, 无论是全球产 量还是需求量都在逐步增加. 甘油作为生物柴油生 产过程的主要副产物, 其产量也在与日俱增 ${ }^{[1-12]}$, 据统计每生产 $1 \mathrm{t}$ 生物柴油大约会产生 $0.1 \mathrm{t}$ 的副产 物 ${ }^{13-16}$. 而甘油本身在食品、医学、涂料、纺织、燃 料等领域就有重要的应用. 生物柴油产业的兴起及 扩大在增加甘油产量的同时也对其市场产生了重大 影响, 导致其价格持续走低 ${ }^{[17]}$. 但从另一个角度来 看, 这也使甘油成为一种理想的、低成本的原料. 将甘油转化成高附加值的化工产品或原料无疑会大 大提高其经济价值, 具有十分可观的发展前景和重 要的现实意义 ${ }^{[3,18-20]}$.

迄今为止，已有多种不同的技术应用于甘油的 转化. 其中, 研究较多的技术是将甘油通过催化选 择性氧化的方式转化为丙烯醛 ${ }^{8,13.21-25]}$ 或丙烯 酸 ${ }^{26-31]}$. 丙烯酸作为重要的化工原料, 广泛应用于 制药、农业、建筑、石油开采等诸多领域 ${ }^{[32-33]}$, 目
前已成为甘油转化利用中最具经济价值和发展前景 的产品, 开发用于该反应的高性能催化剂也成为了 研究热点之一。

目前, 可应用于甘油氧化制丙烯酸的催化剂较 多, 主要包括金属氧化物、杂多酸、分子篮 等 ${ }^{[26.34-41]}$. 但通过研究发现, 杂多酸拥有的酸性位 点过多, 在催化过程中易促进 $\mathrm{CO}_{2}$ 等过度氧化产物 的生成, 大大降低了丙烯酸的选择性和收率. 而分 子篎催化剂则容易产生积碳而使催化剂失活, 显著 降低了催化剂的使用寿命. 与杂多酸和分子篮催化 剂相比, 由于结构中含有的酸性位适量以及独特的 晶格氧氧化优势, 复合金属氧化物催化剂表现出对 丙烯酸较高的选择性和使用过程中更高的热稳定 性. 根据操作过程, 复合金属氧化物催化甘油氧化 制丙烯酸可分为直接法和间接法两种. 直接法具有 设备投资少、操作简单等显著优势, 但对催化剂要 求较高, 间接法工艺成熟、产物收率高, 但设备及 工艺过程较复杂. 我们综述了近年来用于甘油制丙 烯酸所涉及的复合金属氧化物催化剂体系、反应机 理等研究进展, 其中着重阐述复合金属氧化物催化 甘油转化丙烯酸的技术, 分析归纳了该类催化体系

收稿日期: 2020-07-02; 修回日期: 2020-07-21.

基金项目: 国家自然科学基金(21706165); 辽宁省教育厅科学研究项月（LO2019007)；辽宁省自然科学基金计划重点项日(20170540714)

( National Natural Science Foundation of China(21706165); Scientific Research Fund of Liaoning Provincial Education Department, China (LQ2019007); Natural Science Foundation of Liaoning Province, China(20170540714)).

作者简介：卢向轩(1996-)，男，硕士:研究生，(Lu Zi-xuan(1996-), male, master degree candidate).

* 通讯联系人, E-mail: lishuangming@ syuct.edu.cn; E-mail: ssyu@ syuct.edu.cn. 
现存的问题，并对未来的优化和发展方向提出了见 解与展望.

\section{1 复合金属氧化物催化体系}

\section{1 直接法}

直接法是指在同一反应设备中将甘油直接转化 为丙烯酸的一种丁艺方法. 目前直接法所用的复合 金属氧化催化体系主要包括 Mo-V 系、 $W-V(N b)$ 系、 $M_{0}-W-V$ 系和 $F e-V(P)$ 系等. 其中 $M_{0}-V$ 系复合 金属氧化物在H油氧化脱水反应中表现出了优异的 催化活性和丙烯酸选择性, 甘油转化率达 $100 \%$, 丙烯酸选择性最高可达到 $57 \%$ 左右. 其优异的催化 性能得益于拥有适宜的 $\mathrm{V}^{4+} 、 \mathrm{~V}^{5+} 、 \mathrm{~V}-\mathrm{O}$ 键强度和 Brønsted 酸, Mo-V-O 同时提供了氧化还原位点. W$\mathrm{V}(\mathrm{Nb})$ 系催化剂普遍对丙烯酸具有较高的选择性 (最高可达到 60\%), 甘油转化率达 100\%, 其中 WNbO 组分主要催化甘油脱水成丙烯醛, $\mathrm{V}$ 原子几 乎不影响丙烯醛的形成, 但能促进丙烯醛氧化成丙 烯酸. 与前两类不同的是 Fe-V $(P)$ 系虽然对丙烯酸 选择性较低, 但由于 $\mathrm{FeO}_{x}$ 的存在使得该催化剂具有 较高的稳定性.

\subsubsection{Mo- $V$ 系 由于 $M_{0}-V$ 复合金属氧化物中具} 有 $\mathrm{V}_{2} \mathrm{O}_{5}, \mathrm{MoV}_{2} \mathrm{O}_{8}, \mathrm{Mo}_{4} \mathrm{~V}_{6} \mathrm{O}_{25}, \mathrm{MoO}_{3}$ 等多种晶相, 为 了明确 Mo- $\mathrm{V}$ 复合金属氧化物中的关键活性相, 科 研人员做出大量研究工.作. Possato L G 等 ${ }^{42}$ 利用 XRD 对钒和钿前驱体进行热处理时形成的结晶相 进行了原位分析. 研究表明, Mo 和 V 前驱体混合物
在氧气或惰性气氛热处理最终温度 $500{ }^{\circ} \mathrm{C}$ 条件下, 形成的晶相分别为 $\mathrm{MoV}_{2} \mathrm{O}_{8}(61 \%)$ 和 $\mathrm{Mo}_{4} \mathrm{~V}_{6} \mathrm{O}_{25}$ $(29 \%)$. 在H油氧化为丙烯酸的过程中, $\mathrm{MoV}_{2} \mathrm{O}_{8}$ 比 $\mathrm{Mo}_{4} \mathrm{~V}_{6} \mathrm{O}_{25}$ 表现出了更好的丙烯酸选择性, 原因是在 反应期间 $\mathrm{MoV}_{2} \mathrm{O}_{8}$ 中钒的 redox 循环能够产生明显 的动态氧空位, 从而可获得 $97 \%$ 甘油转化率以及 $32 \%$ 丙烯酸选择性. 此外, 作者认为在甘油催化转 化为丙烯酸的过程中, 体系中水蒸气会在催化剂表 面上发生解离并导致 $\mathrm{V}^{5+}$ 位点减少, 导致 $\mathrm{MoV}_{2} \mathrm{O}_{8}$ 向 $\mathrm{Mo}_{4} \mathrm{~V}_{6} \mathrm{O}_{25}$ 转变, 致使催化性能下降. 研究表明, 当 $\mathrm{MoV}_{2} \mathrm{O}_{8} / \mathrm{Mo}_{4} \mathrm{~V}_{6} \mathrm{O}_{25}$ 两种晶相比值为 $1 / 1$ 时表现出了 最佳的催化性能, 且可以通过向反应器中加入纯 $\mathrm{O}_{2}$ 来保持 $\mathrm{MoV}_{2} \mathrm{O}_{8} / \mathrm{Mo}_{4} \mathrm{~V}_{6} \mathrm{O}_{25}$ 活性相的平衡, 从而 提高催化剂的稳定性.

Rasteiro 等 ${ }^{[43]}$ 采用水热合成法制备了一系列 Mo-V-O 催化剂, 并研究了在不同温度和不同气体 流量成分下热处理后的催化性能. 在 $320{ }^{\circ} \mathrm{C}$ 且 $100 \% \mathrm{O}_{2}$ 的流量下进行催化氧化脱水可获得最佳性 能, 丙烯酸选择性为 $33.5 \%$, 甘油转化率为 $100 \%$. 此外, 该研究也发现向反应器中加人 $\mathrm{O}_{2}$ 之后能够 使得含 $\mathrm{V}^{4+}$ 和 $\mathrm{V}^{5+}$ 的氧化物活性相保持平衡. 但由结 果可知, 丙烯酸的选择性并不理想, 这可能与催化 剂较低的比表面积有关. 在此基础上 Rasteiro 等 ${ }^{44}$ 添加 CTAB 和 SDS 表面活性剂合成了纳米棒状 $\mathrm{MoV}_{2} \mathrm{O}_{8}$ 晶相. 结果表明, 表面活性剂的存在可促进 晶相形貌的改变, 从而导致纳米棒状晶体的形成 (图 1). 该催化剂在一步法催化甘油制丙烯酸反应
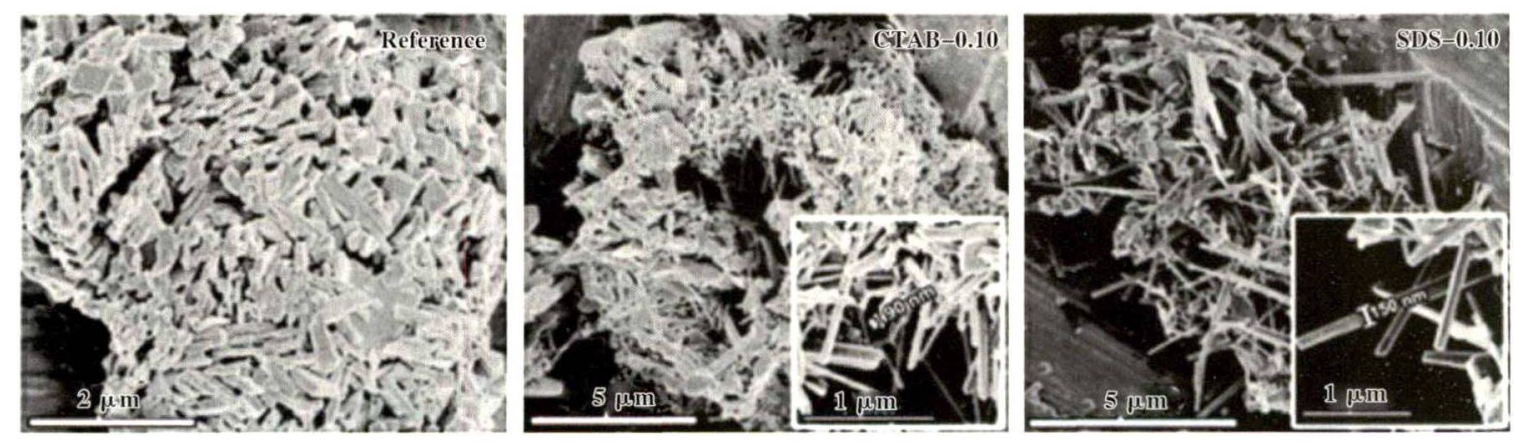

图 1 CTAB 和 SDS 胶束在微晶周围的组织

Fig. 1 Organization of CTAB and SDS micelles around the crystallites ${ }^{4}$

中, 可将甘油完全转化, 且丙烯酸选择性高达 $57 \%$. 其原因在于纳米棒状结构提高了甘油转化过程中的 $\mathrm{MoV}_{2} \mathrm{O}_{8}$ 晶相的稳定性, 避免了钒从晶相迁移到非 晶相, 有利于钒的还原/再氧化, 因此有助于提高
催化性能和寿命.

Shen 等 ${ }^{45}$ 研究了 Mo-V 和 W-V 氧化物催化剂 在空气中将甘油直接氧化为丙烯酸的过程, 详细探 讨了催化剂组分对氧化脱水反应的影响. 研究发 
现, 随着 $\mathrm{W}-\mathrm{V}$ 氧化物催化剂中 $\mathrm{V}$ 含量的增加, $\mathrm{WO}_{3}$ 相从单斜晶变为六方晶和正交晶. 同时, $\mathrm{W}-\mathrm{V}$ 氧化 物催化剂中 $\mathrm{WO}_{3}$ 的微晶尺寸也随着 $\mathrm{V}$ 含量的变化 而明显变化; $\mathrm{Mo}-\mathrm{V}$ 催化剂中的 $\mathrm{Mo}_{6} \mathrm{~V}_{9} \mathrm{O}_{40}$ 晶相具有 较高的催化活性, 可将丙烯醛进一步氧化为丙烯 酸. 分析原因为 $\mathrm{Mo}-\mathrm{V}$ 和 $\mathrm{W}-\mathrm{V}$ 氧化物催化剂中 $\mathrm{V}$ 组 分有利于 $\mathrm{Mo}^{5+}, \mathrm{W}^{5+}$ 和 $\mathrm{V}^{4+}$ 离子的产生, 这些阳离子 的存在会使反应过程中形成更多的活性氧，从而表 现出较高的催化活性. 低 $\mathrm{V}$ 含量的 $\mathrm{Mo}_{1} \mathrm{~V}_{0.25}$ 和 $\mathrm{W}_{1} \mathrm{~V}_{0.25}$ 催化剂在 $300{ }^{\circ} \mathrm{C}$ 反应温度下, 丙烯酸产率分 别高达 $20.1 \%$ 和 $25.7 \%$. 此外, 碳平衡随着反应温 度的降低而增加, 表明较低的反应温度可抑制 W-V 氧化物催化剂在甘油氧化脱水反应中积碳的发生.

1.1.2 W-V $(\mathrm{Nb})$ 系研究认为, W-V 系催化剂之 所以能够将甘油一步法转化为丙烯酸, 是因为催化 剂同时具有 $\mathrm{WO}_{3}$ 的酸性和 $\mathrm{V}$ 离子的氧化性. 为了明 确催化剂最佳的原子配比, Soriano M D 等 ${ }^{[46]}$ 通过 水热法制备了 $\mathrm{W}-\mathrm{V}$ 混合氧化物催化剂. 研究表明, 当 $\mathrm{W} 、 \mathrm{~V}$ 原子比为 $\mathrm{V} /(\mathrm{W}+\mathrm{V})=0.12 \sim 0.21$ 时, 丙 烯酸收率为 $25 \%$. 作者认为该催化剂中由铇氧化物 赋予的酸性以及由 $\mathrm{WO}_{3}$ 晶相内结合 $\mathrm{V}$ 离子赋予的 氧化性共同提供了两种活性位点.

Omata $\mathrm{K}$ 等 ${ }^{[47]}$ 通过水热法添加磷酸制备了 W$\mathrm{V}-\mathrm{Nb}$ 的复合金属氧化物催化剂. 研究表明, 添加磷 酸后丙烯酸产率从 $46.2 \%$ 提升至 $59.2 \%$, 表明添加 磷酸的 $\mathrm{W}-\mathrm{V}-\mathrm{Nb}$ 的复合金属氧化物催化剂可显著提 高甘油直接转化丙烯酸的催化性能. 此外, 添加磷 酸后催化剂酸性位点数量从 129 增加到 $250 \mathrm{~g} /$ $\mu \mathrm{mol}$, 且增加了 Brønsted 和 Lewis 酸度的比率, 因 此作者推测催化性能的改善可能是由于酸量和 Brønsted 酸度的增加. 通过对比还发现, 未添加磷 酸的 W-V-Nb-O 催化剂在高于 $308{ }^{\circ} \mathrm{C}$ 的反应温度 下, 丙烯酸收率急剧下降, 而当采用 $\mathrm{H}_{3} \mathrm{PO}_{4} / \mathrm{W}-\mathrm{V}$ $\mathrm{Nb}-\mathrm{O}$ 催化剂时, 该下降明显地被抑制了, 显然添加 磷酸可以抑制丙烯酸的深度氧化.

在 W-V-O 基础上, Chieregato A 等 ${ }^{[48]}$ 研究了掺 $\mathrm{Nb}$ 的 W $-\mathrm{V}$ 复合氧化物的六方铇青铜结构, 进行了 甘油一锅法氧化制丙烯酸的催化性能研究. 结果表 明, 在三组分六方铇青铜结构中掺人 $\mathrm{Nb}$ 元素获得 了 $34 \%$ 的最佳丙烯酸收率, 远高于未掺杂 $\mathrm{Nb}$ 催化 剂的丙烯酸收率 $(25 \%)$, 同时在寿命实验中也表现 出更高的稳定性, 证实了 $\mathrm{Nb}^{5+}$ 掺杂可增加六方铇青 铜结构的酸性位点. 然而, 随着 $\mathrm{Nb}$ 含量的增加, 丙
烯酸选择性出现了显著的降低，从 $26.5 \%$ 降到 $11 \%$, 这表明少量的 $\mathrm{Nb}$ 有利于形成丙烯酸, 但当 $\mathrm{Nb}$ 掺杂量过高时则会促进 1,3-二氧戊环-4-甲醇等 大分子物质的形成. 此外, 当催化反应连续进行 $100 \mathrm{~h}$ 时, 仅观察到对丙烯酸的选择性略有下降 (从 33\% 34\%下降至 30.5\%), 是由于六方铇青铜结构 中 $\mathrm{V}^{4+}$ 的逐步氧化所致. 作者还发现, 丙烯醛和丙烯 酸的选择性受反应条件的影响很大: 例如当反应温 度高于 $300{ }^{\circ} \mathrm{C}$ 或接触时间超过 $0.15 \mathrm{~s}$ 时便容易生成 $\mathrm{CO}_{x}$ 和其他大分子物质.

Delgado $\mathrm{D}^{\text {等 }}{ }^{\left[{ }^{36]}\right.}$ 水热合成了一系列具有不同 $m$ $\mathrm{WO}_{3}$ 和 $h-\mathrm{WO}_{3}$ 相含量的 W-V-O 催化剂. 研究表明, 含 $\mathrm{V}$ 的 $h-\mathrm{WO}_{3}$ 晶相可促进丙烯醛和丙烯酸的形成, 催化剂中 $h-\mathrm{WO}_{3}$ 相含量较低时, 主要形成碳氧化物 和大分子物质. 含 $\mathrm{V}$ 的 $h-\mathrm{WO}_{3}$ 晶相呈现的 $\mathrm{V}^{5+}$ 位点 表现出比 $m-\mathrm{WO}_{3}$ 晶相更高的还原性. 甘油氧化脱水 反应催化结果表明, $m-\mathrm{WO}_{3}$ 相中不存在酸性位点且 对丙烯酸的形成具有负面影响. 产物中丙烯酸/丙 烯醛比例随 $h-\mathrm{WO}_{3}$ 比例的增加而增加, 这意味着进 人六方铇青铜结构的钒位点对丙烯醛部分氧化为丙 烯酸具有选择性, 而 $m$ - $\mathrm{WO}_{3}$ 在甘油部分氧化中选择 性较低, 主要生成碳氧化物和甘油低聚产物。

与 Mo- $\mathrm{V}$ 复合金属氧化物相比, $\mathrm{W}-\mathrm{V}(\mathrm{Nb})$ 复合 金属氧化物可显著提高丙烯酸产率, 这是由于 $\mathrm{WO}_{3}$ 晶相结合 $\mathrm{V}$ 可将丙烯醛选择性氧化为丙烯酸, 同时 也提高了催化剂的热稳定性. 尽管 $\mathrm{W}-\mathrm{V}(\mathrm{Nb})$ 复合 金属氧化物和其它催化剂在选择性生产丙烯酸方面 展现了优异的性能, 但需要相对稳定的反应温度, 且对 $W-V(N b)$ 复合金属氧化物活性位点仍需进一 步深人研究.

1.1.3 Mo-W-V 系除了 Mo-V、W-V 两种催化剂 体系外, Liu 等 ${ }^{[49]}$ 采用不同的裧烧温度制备了一系 列 $\mathrm{Al}_{2} \mathrm{O}_{3}$ 负载 $\mathrm{Mo}-\mathrm{W}-\mathrm{V}$ 复合金属氧化物催化剂. 研 究表明, 当炦烧温度为 $350{ }^{\circ} \mathrm{C}$ 时, 丙烯醛选择性为 $35.2 \%$ 而丙烯酸选择性为 $19.8 \%$, 通过以上数据我 们发现, 在 $350{ }^{\circ} \mathrm{C}$ 下炦烧的催化剂活性不足以将丙 烯醛转化为丙烯酸; 但当㷽烧温度提高到 $450{ }^{\circ} \mathrm{C}$ 时, 丙烯酸的选择性达到 $46.2 \%$, 而丙烯醛仅为 $3.0 \%$, 同时与 $350{ }^{\circ} \mathrm{C}$ 下㷽烧相比, $\mathrm{CO}_{x}$ 的选择性急 剧提高, 这说明传烧温度的提高不仅促进丙烯酸的 生成而且增强了氧化程度. 当煅烧温度提高到 550 、 $650{ }^{\circ} \mathrm{C}$ 时, 丙烯酸选择性出现了下降, 丙烯醛选择 性则增加了. 为了了解炦烧温度对催化剂晶体结构 
的影响，作者又做了一组对比实验：合成的催化剂 无煅烧和 $350{ }^{\circ} \mathrm{C}$ 䍩烧的样品显示出相同的 XRD 衍 射峰(六边形的 ( V, Mo) $\mathrm{O}_{3}$ 或钿酸铵), 但 $450{ }^{\circ} \mathrm{C}$ 炦 烧的催化剂晶体结构完全不同 (类似于正交 ( V, $\mathrm{Mo}) \mathrm{O}_{3}$ 物种), 与此同时观察到 $\mathrm{MoO}_{3}$ 消失了, 并被
许多复杂的团聚体所取代 ( 例如 $\mathrm{Mo}_{4} \mathrm{O}_{11}, \mathrm{~V}_{2} \mathrm{O}_{5}$, $\mathrm{VO}_{2}$ 以及 $(\mathrm{V}, \mathrm{Mo})_{2} \mathrm{O}_{5}$ 和 $\left.(\mathrm{V}, \mathrm{Mo})_{4} \mathrm{O}_{11}\right)$, 将煅烧温度 进一步提高到 550 和 $650{ }^{\circ} \mathrm{C}$ 会使催化剂中 $\mathrm{Mo}_{3} \mathrm{VO}_{x}$ 和 $\mathrm{WO}_{x}$ 发生连续的结构变化 (图 2). 这很好地解释 了在 550 和 $650{ }^{\circ} \mathrm{C}$ 炦烧时, 丙烯醛选择性的增加以
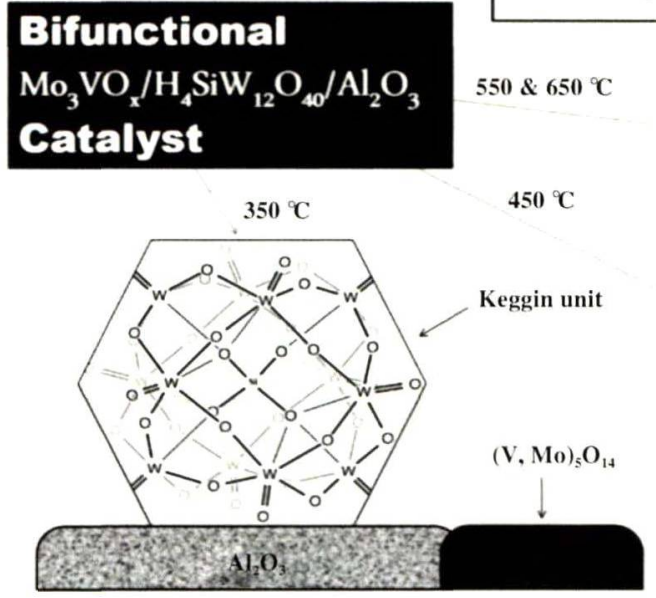

$\mathrm{ACR}=2.9 \%, \mathrm{AA}=12.1 \%$

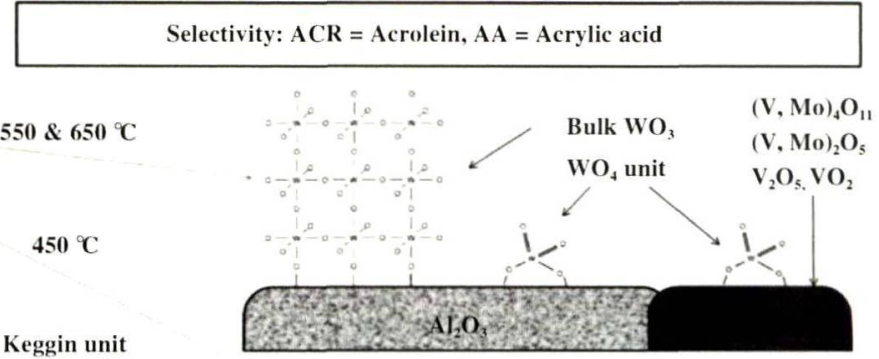

$\mathrm{ACR}=15.4 \%, \mathrm{AA}=5.3 \%$

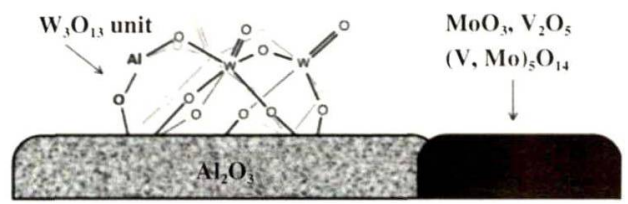

$\mathrm{ACR}=7.8 \%, \mathrm{AA}=11.9 \%$

图 2 不同炈烧温度下负载物种结构演化方案 ${ }^{[49]}$

Fig.2 Scheme of structure evolution of supported species under different calcinations temperature ${ }^{49}$

及丙烯酸选择性的下降, 这是由于高温煅烧后 $(\mathrm{V}$, Mo ) ${ }_{5} \mathrm{O}_{14}$ 活性晶相的消失, 此外催化剂表面积下降 和孔结构的损失也可能导致催化性能的变化.

此外, Yun 等 ${ }^{[50]}$ 通过密度泛函理论计算 (DFT), 将 $\mathrm{W}$ 掺人 Mo- $\mathrm{V}$ 复合氧化物中模拟制备了 双功能 $M_{0}-\mathrm{V}-\mathrm{W}$ 复合氧化物催化剂. 结果表明, $\mathrm{MoV}$ 中的 $\mathrm{Mo}$ 或 $\mathrm{V}$ 被掺人的 $\mathrm{W}^{6+}$ 所替代, 催化剂酸 强度得到显著提升, 有利于甘油氧化脱水反应. 此 外, 研究发现, 掺人的元素以 $W^{6+}$ 为主, $W^{6+}$ 可将 $\mathrm{MoV}$ 晶体中的部分 $\mathrm{Mo}^{6+}$ 和 $\mathrm{V}^{5+}$ 分别还原成 $\mathrm{Mo}^{5+}$ 和 $\mathrm{V}^{4+}$, 这样便增加了低价态元素的含量, 同时形成了 较弱的金属-氧相互作用, 提高了催化剂的氧化活 性位比例, 从而有利于丙烯醛进一步氧化为丙烯 酸; 随着 $\mathrm{W}$ 含量的增加, $\mathrm{Mo}^{5+} /\left(\mathrm{Mo}^{5+}+\mathrm{Mo}^{6+}\right)$ 和 $\mathrm{V}^{4+} /\left(\mathrm{V}^{4+}+\mathrm{V}^{5+)}\right.$ 比值以及催化剂的表面积和酸度均 增加, 显著抑制了 $\mathrm{CO}_{x}$ 的生成(选择性降低 $30.6 \%$ ), 丙烯酸的选择性提高了 $8.8 \%$. 此外, $\mathrm{V}^{4+} /\left(\mathrm{V}^{4+}+\right.$ $\left.\mathrm{V}^{5+}\right)$ 之比是决定丙烯酸和 $\mathrm{CO}_{x}$ 选择性的关键因素, $\mathrm{V}^{4+}$ 有利于将丙烯醛转化为丙烯酸而不是深度氧化 产物, MoVW-5 催化剂在 $250{ }^{\circ} \mathrm{C}$ 下, 丙烯酸选择性 达到 $30.5 \%$. 研究认为 $\mathrm{W}$ 的存在不仅起到酸性物质
的作用，而且还具有促进剂的作用.

\subsubsection{Fe- $V(P)$ 系 $\quad F e-V(P)$ 复合金属氧化物也被} 人们用于甘油催化氧化制丙烯酸的反应中. Wang 等 ${ }^{[51]}$ 通过固态合成法制备了负载 $\mathrm{Fe}_{2} \mathrm{O}_{3}$ 的 $\mathrm{Fe}-\mathrm{V}$ 复 合氧化物催化剂, 并研究了其对甘油脱氧串联反应 的催化作用. $\mathrm{FeVO}_{4}$ 颗粒的表面被 $\mathrm{V}=\mathrm{O}$ 和 $\mathrm{V}-\mathrm{OH}$ 基 团覆盖, 它们分别通过甘油的中碳和末端碳位置处 脱水而生成丙烯醛或着丙酮的活性位点, 生成的丙 烯醛被重新吸附到 $\mathrm{FeO}_{x}$ 上, 从而被进一步氧化为丙 烯酸. 作者认为, 在甘油氧化制丙烯酸的过程中, $\mathrm{FeVO}_{4}$ 相和 $\mathrm{FeO}_{x}$ 结构分别为甘油脱水以及丙烯醛氧 化为丙烯酸提供了相应的活性位点, 且嵌人式结构 可稳定纳米尺寸的 $\mathrm{FeO}_{x}$ 结构, 进一步提高了催化剂 的稳定性.

\section{2 间接法}

甘油间接法制丙烯酸与直接法的区别在于, 甘 油 一内烯醛、丙烯醛 $\rightarrow$ 丙烯酸这两个反应过程是分 别在两个反应设备中完成的, 首先在催化剂 $\mathrm{A}$ 作用 下将甘油催化脱水氧化生成丙烯醛, 然后在催化剂 B 作用下再将丙烯醛进一步氧化为丙烯酸. 与直接 法相比, 间接法的两步串联或耦合使得丙烯酸收率 可达到 $80 \%$ 以上, 从工业应用的角度更具有发展潜 
力. 用于丙烯醛氧化制丙烯酸的催化剂及相关反应 工艺都已经实现了工业化. 因此, 将甘油高选择性 地氧化为丙烯醛是间接法的关键步骤和研究重点. 目前, 用于甘油选择性氧化制丙烯醛的复合金属氧 化物催化剂主要有 $\mathrm{Zr}-\mathrm{Nb}-\mathrm{O} 、 \mathrm{~W}-\mathrm{Nb}-\mathrm{O} 、 \mathrm{Cs}-\mathrm{W}-\mathrm{O}$ 和 Fe-P-O 体系.

Lauriol-Garbay P 等 ${ }^{[52]}$ 合成的 ZrNbO 催化剂既 能有效地将甘油脱水成丙烯醛, 又具有较高的稳定 性, 在运行 $177 \mathrm{~h}$ 后仍显示出 $82 \%$ 的转化效率以及 稳定的丙烯醛选择性. 这是由于 $\mathrm{Nb}$ 含量的增加对 催化剂的稳定性产生了积极的影响. 尽管以往的研 究认为 Brønsted 酸性位点和 Lewis 酸性位点都对反 应起到了积极作用，但同时两种酸的强度也会对反 应产生一定的负面影响, 作者在该研究中就发现, 催化剂的失活与在强 Lewis 酸性位点上形成焦炭有 关. 这一问题可通过在反应过程中提高氮气流速来 解决, 这是由于流速的增加减少积碳前驱体在反应 器中的停留时间从而抑制了积碳的形成. 研究认 为, $\mathrm{ZrNbO}$ 的高稳定性不仅与铌掺人氧化锆中以及 Lewis 和 $\mathrm{ZrO}_{2}$ 载体的碱性位点有关, 同时还与氧化 铌固有性质有关.

Omata 等 ${ }^{[33]}$ 利用水热法合成了具有层状结构 的 W-Nb-O $\left(700{ }^{\circ} \mathrm{C}\right) 、 \mathrm{~W}-\mathrm{Nb}-\mathrm{O}\left(1000^{\circ} \mathrm{C}\right) 、 \mathrm{Cs}-\mathrm{W}-$ $\mathrm{Nb}-\mathrm{O}$ 系列催化剂, 研究了催化剂晶相结构对甘油 脱水制丙烯醛反应选择性的影响. 这 3 种催化剂在 $\mathrm{c}$ 方向上都是八面体层状排列, 但 $\mathrm{a}-\mathrm{b}$ 平面结构不 同, 其中 $\mathrm{W}-\mathrm{Nb}-\mathrm{O}\left(700{ }^{\circ} \mathrm{C}\right)$ 为无序排列, W-Nb-O $\left(1000{ }^{\circ} \mathrm{C}\right.$ ) 基于 $\mathrm{Nb}_{8} \mathrm{~W}_{9} \mathrm{O}_{47}$ 相有序排列, Cs-W-Nb-O 基于正交结构有序排列. 研究表明, 虽然 $\mathrm{W}-\mathrm{Nb}-\mathrm{O}$ $\left(700{ }^{\circ} \mathrm{C}\right)$ 和 Cs-W-Nb-O 具有相近的丙烯醛选择性 (W-Nb-O ( $700{ }^{\circ} \mathrm{C}$ ) $29.7 \%$, Cs-W-Nb-030.5\%), 但 是催化活性却显著不同. 而 $\mathrm{W}-\mathrm{Nb}-\mathrm{O}\left(1000{ }^{\circ} \mathrm{C}\right)$ 和 Cs-W-Nb-O 相比, 脱水产物 (例如羟丙酮) 的产率更 高, 而丙烯醛的产率较低. 研究表明 $\mathrm{W}-\mathrm{Nb}-\mathrm{O}$ 复合 金属氧化物是一种高效且有潜力催化剂，在甘油制 丙烯醛反应中收率高达 70\% 以上.

Wang 等 ${ }^{[54]}$ 通过水热法制备了含有不同晶相
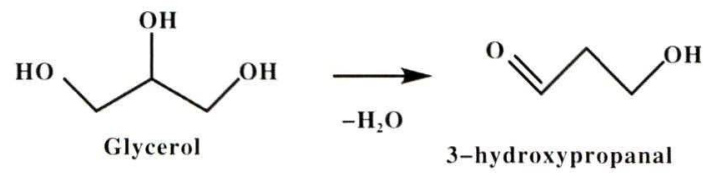

$\left(\mathrm{Cs}_{0.12} \mathrm{WO}_{3}, \mathrm{CsW}_{1.6} \mathrm{O}_{6}, \mathrm{CsW}_{2} \mathrm{O}_{6}\right)$ 的 Cs-W-O 复合金 属氧化物催化剂. 研究发现, 纯相 Cs-W-O 复合金 属氧化物对甘油脱水制丙烯醛具有良好的催化效 果, 且铯的存在提高了丙烯醛的选择性. 当 $\mathrm{Cs} / \mathrm{W}$ 摩尔比为 $0.2 / 1$ 时, 制备的 Cs-W-O 具有最好的催 化性能, 甘油转化率为 $81.3 \%$, 丙烯醛的选择性达 到 $92.9 \%$.

Deleplanque 等 ${ }^{[26]}$ 对合成的 $\mathrm{FePO}_{4}$ 催化甘油脱 水进行了研究, 发现 $\mathrm{FePO}_{4}$ 对丙烯醛具有较高的选 择性. 研究通过水热合成、沉淀-浓缩法、氨法以及 氨水热法均制备出了 $\mathrm{FePO}_{4}$ 催化剂, 通过对比发现 用氨水热法合成的磷酸铁催化剂 ( FeP-P 和 FeP-H) 呈现出形状相对良好的颗粒, 具有大而平坦的表面 $\left(43.6 \mathrm{~m}^{2} / \mathrm{g}\right)$. 同时水热合成法也能得到晶型结构良 好的纯单斜晶 $\mathrm{FePO}_{4}$ 催化剂, 且稳定性更高. $\mathrm{FeP}-\mathrm{H}$ 在 $280^{\circ} \mathrm{C}$ 下催化反应 $5 \mathrm{~h}$, 甘油可完全转化, 并且对 丙烯醛选择性高达 $92 \%$ 。

\section{2 催化甘油氧化反应机理}

研究认为, 甘油脱水氧化生成丙烯酸需要 3 步 反应(路线图 1): 第 1 步, 甘油分子在催化剂的酸 性位点 ( $\mathrm{B}$ 和 $\mathrm{L}$ 酸) 上发生脱水反应生成 3-羟基丙 醛; 第 2 步, 3-羟基丙醛再经脱水生成中间产物丙 烯醛, 最后丙烯醛发生氧化生成丙烯酸. 甘油脱水 氧化机理如图 3 所示, 由于甘油分子中同时存在端 位羟基和仲位羟基，当在 $\mathrm{B}$ 和 $\mathrm{L}$ 酸位发生脱水时, 会分别生成 3-羟基丙醛和羟基丙酮. 3-羟基丙醛再经 脱水可形成丙烯醛, 并且通过逆羟醛缩合反应, 3-差 基丙醛还产生乙醛和甲醛. 甲醛可与甘油反应形成环 醚产物, 或与氧气反应形成甲酸和 $\mathrm{CO}_{x}$. 如果催化剂 表面发生积碳, 反应则更倾向于生成羟基丙酮、乙醛 和丙醛等产物, 并在有氧条件下继续生成甲酸、乙 酸、丙酸和 $\mathrm{CO}_{x}$. 通过反应机理可知, 对于甘油选择 性氧化制丙烯酸反应而言, 理想的催化剂不仅应当 拥有合适的酸性位点和氧化还原位点, 还应在催化 过程中具有抑制积碳发生的结构特点, 这样才能获 得高收率的丙烯酸以及持续的稳定性 ${ }^{[26,51]}$.

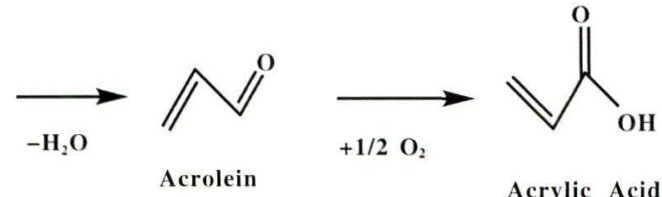

图示 1 甘油三步转化为丙烯酸

Scheme 1 The three steps required for glycerol transformation into acrylic acid 


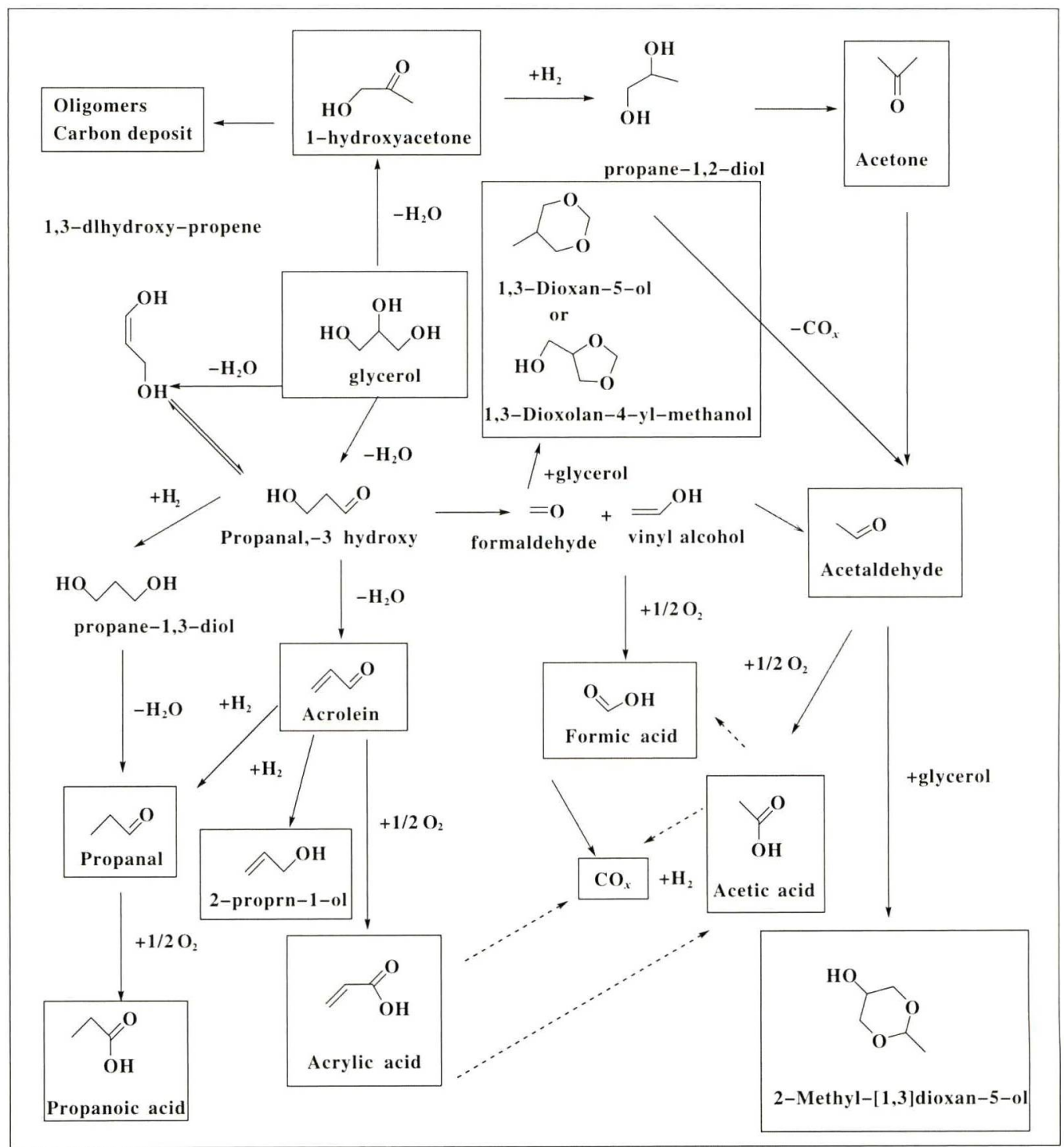

图 3 甘油氧化脱水反应机理示意图 ${ }^{[26]}$

Fig.3 Proposed mechanism of (oxy) dehydration of glycerol ${ }^{[26]}$

基于对甘油氧化反应的认识, 随后的研究进一 步拓展了对机理的认知, 提出了 Mo-V - O 和 W-V - O 复合金属氧化物催化甘油氧化的反应机理. Shen 等 ${ }^{[45]}$ 从催化剂活性位点 (例如 $\mathrm{Mo}^{5+} 、 \mathrm{~W}^{5+}$ 和 $\mathrm{V}^{4+}$ ) 的 角度出发, 提出了如图 4 所示的反应路线. 与前述反 应路线一致的是, 作者也认为甘油的质子化是发生 在伯羟基或仲羟基上，前者通过裂解可产生乙醛，而 后者通过两步脱水反应可生成丙烯醛. 丙烯醛再按照 Marsvan-Krevelen 机制进一步氧化为丙烯酸. Drochner 等 ${ }^{[5]}$ 通过研究发现, 丙烯醛羰基中独立电子对与钼 阳离子之间存在相互作用, 这会导致 C- $\mathrm{H}$ 键发生极 化并在之后氧化还原反应中形成配位化合物, 紧接 着羰基化合物被转化为丙烯酸酯, 形成的丙烯酸酯
通过分解进而得到丙烯酸. 这一理论很好地解释了丙 烯醛和乙醛氧化为丙烯酸和乙酸的原因. 此外, 丙烯 酸或乙酸可被进一步氧化而形成 $\mathrm{CO}$ 和 $\mathrm{CO}_{2}$.

\section{3 工艺条件对催化过程的影响}

对于一个理想的催化过程而言, 其技术核心是 高性能的催化剂, 催化剂的晶相结构、表面酸碱 性、氧化还原性以及元素价态等微观结构对催化结 果具有决定性的影响. 在此基础上, 适宜的工艺条 件则可以使催化剂发挥出更佳的催化效果. 表 1 、 表 2 总结了近年来甘油直接法、间接法制丙烯酸反 应中, 各复合金属氧化物催化剂在不同条件下 (反 应温度、接触时间、氧气浓度等) 的催化性能结果. 


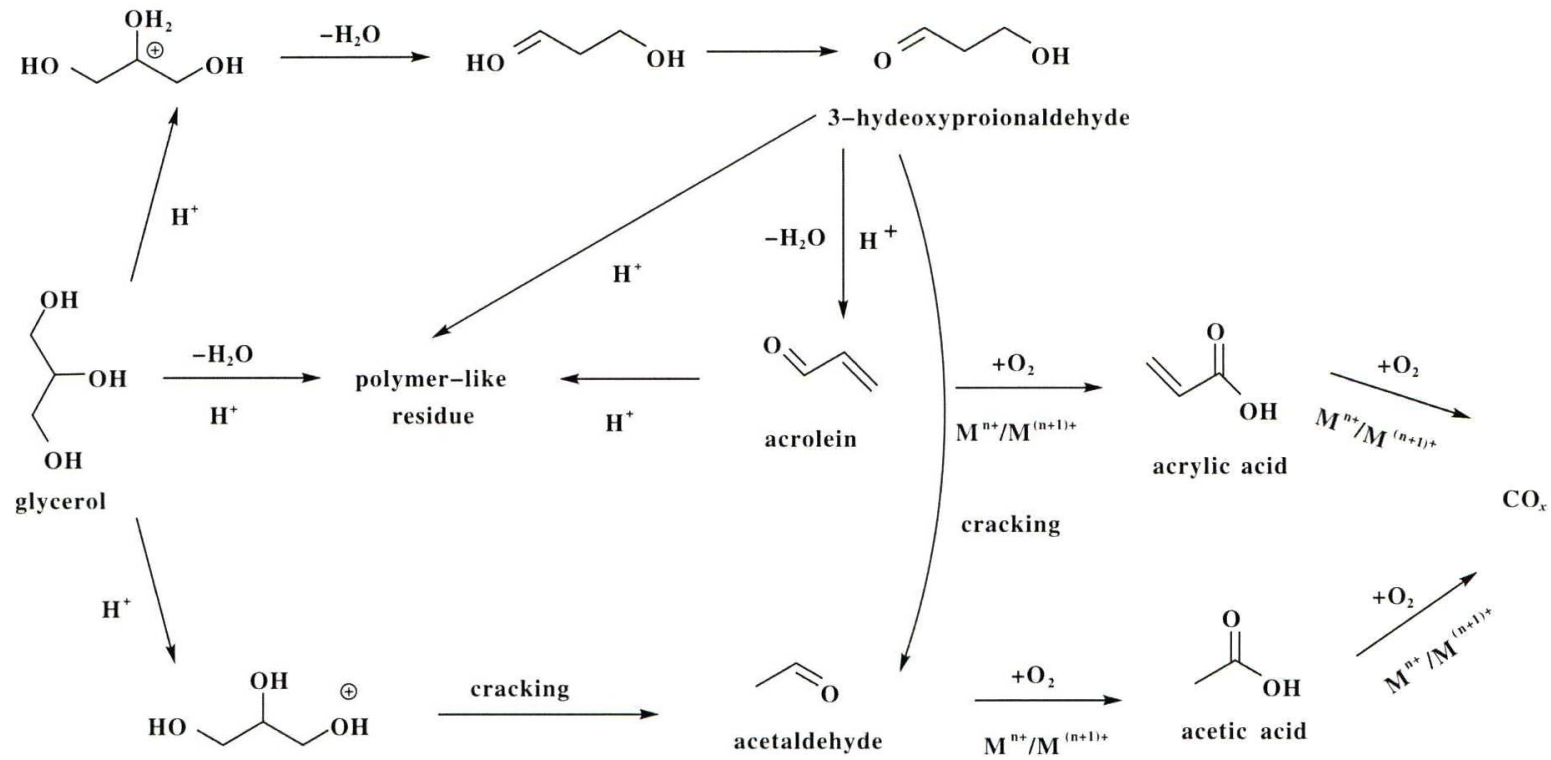

图 $4 \mathrm{Mo} / \mathrm{V}$ 和 $\mathrm{W} / \mathrm{V}$ 氧化物催化剂甘油氧化脱水反应路线 ${ }^{[45]}$

Fig.4 Reaction routes in glycerol oxide hydration over $\mathrm{Mo} / \mathrm{V}$ and $\mathrm{W} / \mathrm{V}$ oxide catalysts ${ }^{[45}$

表 1 复合金属氧化物在不同反应条件下催化甘油选择性氧化结果

Table 1 Results of selective oxidation of glycerol catalyzed by composite metal oxides under different reaction conditions

\begin{tabular}{|c|c|c|c|c|c|c|c|}
\hline Catalyst & $\begin{array}{c}\mathrm{T} \\
/{ }^{\circ} \mathrm{C}\end{array}$ & $\begin{array}{c}\text { Reactant gas } \\
\mathrm{N}_{2} / \mathrm{O}_{2} / \mathrm{H}_{2} \mathrm{O} / \text { glycerol }\end{array}$ & $\begin{array}{c}\text { TOS } \\
/ \mathrm{h}\end{array}$ & $\begin{array}{l}\text { WHSV } \\
/ \mathrm{h}^{-1}\end{array}$ & $\begin{array}{l}\text { Conversion of } \\
\text { glycerol } \%\end{array}$ & $\begin{array}{l}\text { Selectivity to } \\
\text { acrylic acid } / \%\end{array}$ & Ref. \\
\hline $\mathrm{MoVO}$ & 320 & $0 / 28 / 70 / 2$ & 1 & 0.5 & 100 & 57 & [44] \\
\hline WVNbO & 300 & $54 / 4 / 40 / 2$ & 2 & 3.8 & 100 & 34 & [48] \\
\hline WVO & 348 & $54 / 4 / 40 / 2$ & 2 & 0.6 & 100 & 25 & [46] \\
\hline $\mathrm{FeVO}$ & 300 & $66 / 2 / 30 / 2$ & 10 & 20 & 100 & 14 & {$[51]$} \\
\hline MoVWO & 290 & $42 / 12 / 40 / 6$ & 69 & 3.4 & 100 & 42 & {$[35]$} \\
\hline MoVTeNbO & 300 & $23 / 8 / 61 / 8$ & 2 & 0.48 & 99.6 & 28.4 & {$[26]$} \\
\hline
\end{tabular}

表 2 复合金属氧化物在不同反应条件下催化甘油脱水结果

Table 2 Dehydration of glycerol catalyzed by complex metal oxides under different reaction conditions

\begin{tabular}{|c|c|c|c|c|c|c|c|}
\hline Catalyst & $\begin{array}{c}\mathrm{T} \\
1{ }^{\circ} \mathrm{C}\end{array}$ & $\begin{array}{c}\text { Reactant gas } \\
\mathrm{N}_{2} / \mathrm{O}_{2} / \mathrm{H}_{2} \mathrm{O} / \text { glycerol }\end{array}$ & $\begin{array}{c}\text { TOS } \\
/ \mathrm{h}\end{array}$ & $\begin{array}{l}\text { WHSV } \\
/ \mathrm{h}^{-1}\end{array}$ & $\begin{array}{l}\text { Conversion of } \\
\text { glycerol } / \%\end{array}$ & $\begin{array}{l}\text { Selectivity to } \\
\text { acrylic acid } / \%\end{array}$ & Ref. \\
\hline $\mathrm{ZrNbO}$ & 300 & $51.4 / 0 / 46.3 / 2.3$ & 3 & 3.8 & 100 & 72 & {$[52]$} \\
\hline WNbO & 300 & $44 / 11.2 / 20 / 4$ & 4 & 0.9 & 97.9 & 74.4 & {$[53]$} \\
\hline $\begin{array}{c}\mathrm{Mo}_{3} \mathrm{VO}_{x} / \mathrm{H}_{4} \mathrm{SiW}_{12} \mathrm{O}_{40} / \\
\mathrm{Al}_{2} \mathrm{O}_{3}\end{array}$ & 450 & $59 / 4.2 / 35.1 / 1.7$ & 6 & 10 & 100 & 54.3 & {$[50]$} \\
\hline $\mathrm{FePO}_{4}$ & 300 & $46 / 0 / 48 / 6$ & 5 & 0.48 & 100 & 80 & {$[26]$} \\
\hline
\end{tabular}

\section{1 反应温度}

在反应过程中，甘油首先通过预热器气化，然 后进人反应器进行反应. 通过表 1 可知, 反应温度 在 $290 \sim 350{ }^{\circ} \mathrm{C}$ 范围内. 由于反应要经过脱水和氧化 两大过程，故而温度的控制在整体反应中显得极为
重要, 温度过低导致脱水反应不完全, 中间产物丙 烯醛产率大大降低; 但温度过高, 则会促使丙烯酸 发生 $\mathrm{C}-\mathrm{C}$ 键断裂转而生成 $\mathrm{CO}_{x}$ 等其他副产物 ${ }^{[56]}$. Miller 等 ${ }^{[57]}$ 通过 ${ }^{13} \mathrm{C}$ 同位素示踪剂对丙烯酸 $\mathrm{C}-\mathrm{C}$ 键断裂进行了深人研究, 研究发现键断裂后丙烯酸 
先生成乙烯, 然后在 $\mathrm{MoVO}_{x}$ 催化剂上乙烯被氧化为 乙酸. Rasteiro 等 ${ }^{[33]}$ 在研究 Mo-V-O 催化甘油转化 为丙烯酸性能时得到最有效的温度为 $320^{\circ} \mathrm{C}$, 通过 对比其他温度发现, 在 $290{ }^{\circ} \mathrm{C}$ 下, 丙烯酸更多地吸 附在催化剂表面上, 导致进一步的氧化生成 $\mathrm{CO}_{x}$, 从而很好的说明丙烯酸产率为何降低; 将温度升高 至 $320^{\circ} \mathrm{C}$, 有助于丙烯酸从催化剂表面解吸, 故而 得到最佳的催化效果; 当温度升高至 $350{ }^{\circ} \mathrm{C}$, 在脱 水成丙烯醛之前, 甘油的一部分会分解为 $\mathrm{CO}_{x}$, 所 以中间产物丙烯醛产率大大降低.

\section{2 接触时间}

对于连续催化氧化反应而言, 接触时间过长, 虽然促进了甘油在酸性位点转化为丙烯醛, 但却降 低了丙烯醛在氧化位点上转化为丙烯酸的选择性; 反之, 如果接触时间过短, 则会降低甘油和丙烯醛 与酸性位点以及氧化活性位点接触机会. 因此, 反 应物 (甘油) 和中间体 (丙烯醛) 与催化剂的接触时 间都对反应具有重要的影响. 为了明晰接触时间对 反应的具体影响, Yun 等 ${ }^{[50]}$ 研究发现, 接触时间在 $0.05 \mathrm{~s}$ 下检测到的产物有丙烯醛、乙醛、丙烯酸、 乙酸以及氧化产物 $\left(\mathrm{CO}\right.$ 和 $\left.\mathrm{CO}_{2}\right)$, 这说明即使在很 短的接触时间内甘油也完成了转化. 当接触时间增 加至 $0.16 \mathrm{~s}$ 时, 丙烯酸的选择性从 $22.2 \%$ 增加到 $28.7 \%, \mathrm{CO}_{x}$ 的产率从 $42.5 \%$ 减少到 $37.1 \%$, 该数据 直观地说明当反应物和中间体缺乏与各个活性位点 接触时间时, 会促进副产物生成. 另一方面, 当接 触时间为 $0.16 \mathrm{~s}$ 时, 平衡了脱水和丙烯醛的选择性 氧化, 故而提高了丙烯酸收率, 降低了副产物收率. 随着接触时间的增加 (>0.2 s), $\mathrm{CO}_{x}$ 的选择性显著 增加, 在 $0.27 \mathrm{~s}$ 时达到 $53.1 \%$. 检测时未发现丙烯 醛、乙醛, 但乙酸的含量增加, 进一步说明接触时 间过长, 促进了初级产物向次级产物的转化, 导致 了深度氧化的发生.

\section{3 氧气浓度}

丙烯醛首先在酸性位点上形成, 然后通过钒原 子迅速氧化为丙烯酸, 但是 $\mathrm{V}^{5+}$ 容易被氧化为 $\mathrm{V}^{4+}$, 因此实验室多采用向反应器中通人 $\mathrm{O}_{2}$ 使其得以恢 复. 通过以往研究发现钒的动态氧化还原 $\left(\mathrm{V}^{5+} \rightleftharpoons\right.$ $\mathrm{V}^{4+}$ ) 和丙烯醛的氧化都是根据 Mars-van-Krevelen 机 理进行的, 因此氧气的进料和氧化还原循环密切相 关. 另一方面, 由于 $\mathrm{Mo}^{6+}$ 与 $\mathrm{V}^{4+}$ 相似, 因此在调节晶 格中的钒原子以及改善催化过程中钒氧化态的动态 变化和氧空位的形成方面也具有重要作用. 就这一
问题 Rasteiro 等 ${ }^{[43]}$ 进行了对比实验, 当气流由 $100 \% \mathrm{O}_{2}$ 组成时, 对丙烯酸的选择性最高, 当使用 $100 \% \mathrm{~N}_{2}$ 时, 则不会形成丙烯酸且观察到较少的 $\mathrm{CO}_{x}$ 生成, 这说明缺乏氧化气氛时会阻止产物完全 氧化. 但是当反应气氛中存在 $\mathrm{O}_{2}$ 时, 氧化产物随之 增加; $\mathrm{O}_{2}$ 含量越高, 形成的 $\mathrm{CO}_{x}$ 就越多. Chieregato 等 ${ }^{[58]}$ 通过对比实验发现, 当氧气和甘油的摩尔比 为 $12: 6$ 时可获得最高的丙烯酸选择性 (39\%). 当 氧浓度过低时, 甘油转化率仅 $50 \%$, 并且对丙烯醛 和丙烯酸的选择性都非常低, 相反当氧浓度过高, 丙烯醛等易发生过度氧化生成 $\mathrm{CO}_{x}$, 降低了丙烯酸 选择性.

\section{4 结论与展望}

在当前以及将来很长一段时间内, 生物质资源 利用及相关技术的开发都将会是世界各国重点研究 及发展的内容之一. 而生物柴油则是生物质资源利 用领域最具代表性且发展最快的液体燃料之一，对 该生产过程的主要副产物甘油进行高效利用同样是 重要且迫切的. 复合金属氧化物是催化甘油氧化制 备高附加值产品丙烯酸反应中具有发展前景的催化 剂, 相对于其他催化剂, 其催化效果好、稳定性更 高. 然而, 无论是直接法还是间接法, 进一步研发 具有高选择性及高稳定性的新型复合金属氧化物催 化剂仍是推动生产工艺进步以及该领域发展的 关键.

首先需要解决的是催化剂失活问题, 如常用的 Mo-V-O 催化剂体系中的有效活性组分 $\left(\mathrm{MoV}_{2} \mathrm{O}_{8}\right)$ 极 易被氧化成 $\mathrm{Mo}_{4} \mathrm{~V}_{6} \mathrm{O}_{25}$ 非活性相, 以及 $\mathrm{W}-\mathrm{V}-\mathrm{O}$ 催化 剂体系中 $\mathrm{V}^{4+}$ 的逐步氧化, 这些都能造成催化剂性 能的下降, 大大缩短其使用寿命. 通过向现有晶相 中掺人合适的杂原子进而稳定活性晶相的结构或许 是一种值得深人研究的手段. 另外, 对于甘油氧化 制丙烯酸反应来说, 复合金属氧化物催化剂表面的 酸碱特性以及氧化还原性是影响催化性能优劣的重 要因素, 开发新的制备技术并通过制备过程对催化 剂酸碱性及氧化还原性进行定向调控, 进而实现甘 油分子向丙烯酸分子高选择性的转化同样是今后研 究的主要方向.

\section{参考文献:}

[1] Bechthold I, Bretz K, Kabasci S, et al. Succinic acid: A new platform chemical for biobased polymers from renew- 
able resources [J]. Chem Eng Technol, 2008, 31(5): $647-654$.

[2] Khanna S , Goyal A , Moholkar V S. Production of $n$-butanol from biodiesel derived crude glycerol using clostridium pasteurianum immobilized on amberlite $[\mathrm{J}]$. Fuel, 2013, 112: 557-561.

[3] Behr A, Eilting J, Irawadi K, et al. Improved utilisation of renewable resources: New important derivatives of glycerol $[\mathbf{J}]$. Green Chem, 2008, 10(1): 13-30.

[4] Dimitratos N, Lopez-Sanchez J A, Hutchings G J. Greencatalysis with alternative feedstocks $[\mathrm{J}]$. Top Catal, 2009, 52(3) : 258-268.

[5] Besson M, Gallezot P, Pinel C. Conversion of biomass into chemicals over metal catalysts[J]. Chem Rev, 2014, $114(3): 1827-1870$.

[6] Hu L, Zhao G, Hao W, et al. Catalytic conversion of biomass-derived carbohydrates into fuels and chemicals via furanic aldehydes[J]. RSC Adv, 2012, 2(30) : 1118411206.

[7] Wang Y, Xiao Y, Xiao G. Sustainable value-added C3 chemicals from glycerol transformations: A mini review for heterogeneous catalytic processes $[\mathrm{J}]$. Chin J Chem Eng, 2019, 27(7): 1536-1542.

[8] Katryniok B, Paul S, Capron M, et al. Towards the sustainable production of acrolein by glycerol dehydration [J]. ChemSusChem, 2009, 2( 8) : 719-730.

[9] De Martino M T, Abdelmohsen L, Rutjes F, et al. Nanoreactors for green catalysis $[\mathrm{J}]$. Beilstein J Org Chem, 2018, 14: 716-733.

[10] Zhou C H, Xia X, Lin C X, et al. Catalytic conversion of lignocellulosic biomass to fine chemicals and fuels $[\mathrm{J}]$. Chem Soc Rev, 2011, 40(11): 5588-5617.

[11] Silitonga A S, Atabani A E, Mahlia T M I, et al. A review on prospect of Jatropha curcas for biodiesel in Indonesia[J]. Renew Sust Energ Rev, 2011, 15( 8) : 37333756.

[12] Oh P P, Lau H L N, Chen J, et al. A review on conventional technologies and emerging process intensification (PI) methods for biodiesel production $[\mathrm{J}]$. Renew Sust Energy Rev, 2012, 16(7): 5131-5145.

[13] Katryniok B, Paul S, Dumeignil F. Recent developments in the field of catalytic dehydration of glycerol to acrolein [J]. ACS Catal, 2013, 3(8): 1819-1834.

[14] Ayoub M, Abdullah A Z, Ahmad M, et al. Performance of lithium modified zeolite $\mathrm{Y}$ catalyst in solvent-free conversion of glycerol to polyglycerols [J]. J Taibah Univ Sci, 2018, 8(3) : 231-235.
[15] Yaakob Z , Mohammad M , Alherbawi M , et al. Overview of the production of biodiesel from waste cooking oil [J]. Renewable \& Sustainable Energy Reviews, 2013, 18: $184-193$.

[16] Jerzykiewicz M, Cwielag I, Jerzykiewicz W. The antioxidant and anticorrosive properties of crude glycerol fraction from biodiesel production $[\mathrm{J}]$. J Chem Technol Biot, 2009, 84(8): 1196-1201.

[17] Callam C S, Singer S J, Lowary T L, et al. Computational analysis of the potential energy surfaces of glycerol in the gas and aqueous phases: Effects of level of theory, basis set, and solvation on strongly intramolecularly hydrogen-bonded systems[J]. J Am Chem Soc, 2001, 123 (47): 11743-11754.

[18] Zhou C H, Beltramini J N, Fan Y X, et al. Chemoselective catalytic conversion of glycerol as a biorenewable source to valuable commodity chemicals $[\mathbf{J}]$. Chem Soc Rev, 2008, 37(3) : 527-549.

[19] Viswanadham B, Pavankumar V, Chary K V R. Vaporphase dehydration of glycerol to acrolein over phosphotungstic acid catalyst supported on niobia[J]. Catal Lett, 2014, 144(4) : 744-755.

[20] Sun D, Yamada Y, Sato S, et al. Glycerol as a potential renewable raw material for acrylic acid production $[\mathbf{J}]$. Green Chem, 2017, 19(14) : 3186-3213.

[21] Foo G S, Wei D, Sholl D S, et al. Role of Lewis and Brønsted acid sites in the dehydration of glycerol over niobia[J]. ACS Catal, 2014, 4(9) : 3180-3192.

[22] Mahdavi V, Monajemi A. Gas phase dehydration of glycerol catalyzed by gamma $\mathrm{Al}_{2} \mathrm{O}_{3}$ supported $\mathrm{V}_{2} \mathrm{O}_{5}$ : A statistical approach for simultaneous optimization $[\mathrm{J}]$. RSC Adv, 2016, 6(115): 114244-114255.

[23] Denkwitz Y, Zhao Z, HRmann U , et al. Stability and deactivation of unconditioned $\mathrm{Au} / \mathrm{TiO}_{2}$ catalysts during $\mathrm{CO}$ oxidation in a near-stoichiometric and $\mathrm{O}_{2}$-rich reaction atmosphere $[J]$. J Supercrit Fluid, 2007, 251(2): 363-373.

[24] Sung $\mathrm{K} \mathrm{H}$, Cheng $\mathrm{S}$. Effect of $\mathrm{Nb}$ doping in $\mathrm{WO}_{3} / \mathrm{ZrO}_{2}$ catalysts on gas phase dehydration of glycerol to form acrolein[J]. RSC Adv, 2017, 7(66) : 41880-41888.

[25] Yu W, Wang P, Zhou C, et al. Acid-activated and WOloaded montmorillonite catalysts and their catalytic behaviors in glycerol dehydration[J]. Chin J Catal, 2017, 38 (6) : 1087-1100.

[26] Deleplanque J, Dubois J L, Devaux J F, et al. Production of acrolein and acrylic acid through dehydration and oxydehydration of glycerol with mixed oxide catalysts $[\mathrm{J}]$. 
Catal Today, 2010, 157(1/4): 351-358.

[27] Thanasilp S, Schwank J W, Meeyoo V, et al. Preparation of supported POM catalysts for liquid phase oxydehydration of glycerol to acrylic acid $[\mathbf{J}] . J$ Mol Catal AChem, 2013, 380 : 49-56.

[28] Witsuthammakul A, Sooknoi T. Direct conversion of glycerol to acrylic acid via integrated dehydration-oxidation bed system $[\mathrm{J}]$. Appl Catal A-Gen, 2012, 413/414: $109-116$

[29] Sarkar B, Pendem C, Sivakumar Konathala L N, et al. $\mathrm{Cu}$ nanoclusters supported on nanocrystalline $\mathrm{SiO}_{2}$ $\mathrm{MnO}_{2}$ : A bifunctional catalyst for the one-step conversion of glycerol to acrylic acid $[\mathrm{J}]$. Chem Commun, 2014, 50 (68) : 9707-9710.

[30] Wang F, Dubois J L, Ueda W. Catalytic selydration of glycerol over vanadium phosphate oxides in the presence of molecular oxygen [J]. J Catal, 2009, 268(2) : 260267.

[31] Wu S T, She Q M, Tesser R, et al. Catalytic glycerol dehydration-oxidation to acrylic acid $[\mathrm{J}]$. Catal Rev, ( 2020 ), doi : 10.1080/01614940.2020.1719611.

[32] Feng X, Sun B, Yao Y, et al. Renewable production of acrylic acid and its derivative: New insights into the aldol condensation route over the vanadium phosphorus oxides [J]. J Catal, 2014, 314: 132-141.

33] Xu X, Lin J, Cen P. Advances in theresearch and development of acrylic acid production from biomass $[\mathrm{J}]$. Chin J Chem Eng, 2006, 14(4) : 419-427.

[34] Paula A S, Possato L G, Ratero D R, et al. One-step oxide hydration of glycerol to acrylic acid using ETS-10-like vanadosilicates [J]. Micropor Mesopor Mat, 2016, 232 : $151-160$.

[35] Chieregato A, Soriano M D, Garcia-Gonzalez E, et al. Multielement crystalline and pseudocrystalline oxides as efficient catalysts for the direct transformation of glycerol into acrylic acid $[\mathbf{J}]$. ChemSusChem, 2015, 8(2) : 398406.

[36] Delgado D, Chieregato A, Soriano M D, et al. Influence of phase composition of bulk tungsten vanadium oxides on the aerobic transformation of methanol and glycerol $[\mathrm{J}]$. Eur J Inorg Chem, 2018, 2018(10): 1204-1211.

[37] Possato L G, Diniz R N, Garetto T, et al. A comparative study of glycerol dehydration catalyzed by micro/mesoporous MFI zeolites[J]. J Catal, 2013, 300: 102-112.

[38] Liu Rong(刘蓉), Xiao Tian-cun(肖天存), Wang Xiao-long (王晓龙), et al. Preparation of acrylic acid from biomass by-product crude glycerol (生物质副产物粗H
油制备丙烯酸) [J]. Petrkchem Technol (石油化工), 2018, . 820-826.

[39] Chen 、i a(陈小娜), He Dan-dan(何丹丹), Chen Z: (陈志鹏), et al. Preparation of acrylic acid by calalytic oxidative dehydration of crude glycerol by-product of biodiesel (生物柴油副产物粗甘油催化氧化脱水 制备丙烯酸) [ J ] . Chin J Process Eng (过程工程学 报), 2019, 2019(S01): 123-128

40 ] Possato L G, Acevedo M D, Padró C L, et al. Activation of Mo and V oxides supported on ZSM-5 zeolite catalysts followed by in situ XAS and XRD and their uses in oxydehydration of glycerol [J]. Mol Catal, 2020, 481 : 110158.

[41] Dos Santos M B, Andrade H M C, Mascarenhas A J S. Oxidative dehydration of glycerol over alternative $\mathrm{H}$, FeMCM-22 catalysts: Sustainable production of acrylic acid [J]. Micropor Mesopor Mat, 2019, 278: 366-377.

[42] Possato L G, Cassinelli W H, Meyer C I, et al. Thermal treatments of precursors of molybdenum and vanadium oxides and the formed $\mathrm{Mo}_{x} \mathrm{~V}, \mathrm{O} z$ phases active in the oxydehydration of glycerol $[\mathrm{J}]$. Appl Catal A-Gen, 2017, 532: $1-11$.

[43] Rasteiro L F, Vieira L H, Possato L G, et al. Hydrothermal synthesis of Mo- $V$ mixed oxides possessing several crystalline phases and their performance in the catalytic oxydehydration of glycerol to acrylic acid $[\mathrm{J}]$. Catal Today, 2017, 296: 10-18.

[44] Rasteiro L F, Vieira L H, Santilli C V, et al. Surfactantassisted synthesis of Mo-V mixed oxide catalysts for upgraded one-step conversion of glycerol to acrylic acid $[\mathrm{J}]$. RSC Adv, 2018, 8(22) : 11975-11982.

[45] Shen L, Yin H, Wang A, et al. Gas phase oxidehydration of glycerol to acrylic acid over $\mathrm{Mo} / \mathrm{V}$ and $\mathrm{W} / \mathrm{V}$ oxide catalysts [J]. Chem Eng J, 2014, 244: 168-177.

[46] Soriano M D, Concepción P, Nieto J M L, et al. Tungsten-Vanadium mixed oxides for the oxidehydration of glycerol into acrylic acid $[\mathrm{J}]$. Green Chemistry, 2011, 13 (10) : 2954-2962.

[47] Omata K, Matsumoto K, Murayama T, et al. Directoxidative transformation of glycerol into acrylic acid over phosphoric acid-added $\mathrm{W}-\mathrm{V}-\mathrm{Nb}$ complex metal oxide catalysts [J]. Chem Lett, 2014, 43(4): 435-437.

[48] Chieregato A, Basile F, Concepción P, et al. Glycerol oxidehydration into acrolein and acrylic acid over W-VNb-O bronzes with hexagonal structure $[\mathbf{J}]$. Catal Today, 2012, 197(1) : 58-65.

[49] Liu L, Wang B, Du Y, et al. Bifunctional $\mathrm{Mo}_{3} \mathrm{VO}_{x} / \mathrm{H}_{4}$ - 
$\mathrm{SiW}_{12} \mathrm{O}_{40} / \mathrm{Al}_{2} \mathrm{O}_{3}$ catalysts for one-step conversion of glycerol to acrylic acid: Catalyst structural evolution and reaction pathways $[\mathrm{J}]$. Appl Catal B-Environ, 2015, 174/ 175: $1-12$.

[50] Yun Y S, Lee K R, Park H, et al. Rationaldesign of a bifunctional catalyst for the oxydehydration of glycerol: A combined theoretical and experimental study $[\mathrm{J}]$. ACS Catal, 2014, 5(1) : 82-94.

[51] Wang F, Xu J, Dubois J L, et al. Catalytic oxidative dehydration of glycerol over a catalyst with iron oxide domains embedded in an iron orthovanadate phase $[\mathrm{J}]$. ChemSusChem, 2010, 3(12): 1383-1389.

[52] Lauriol-Garbay P, Millet J M M, Loridant S, et al. New efficient and long-life catalyst for gas-phase glycerol dehydration to acrolein $[J]$. J Catal, 2011, 280(1): 6876.

[53] Omata K, Izumi S, Murayama T, et al. Hydrothermal synthesis of $\mathrm{W}-\mathrm{Nb}$ complex metal oxides and their application to catalytic dehydration of glycerol to acrolein $[\mathrm{J}]$. Catal Today, 2013, 201: 7-11.

[54] Wang Ting-ting (王婷婷), Song Yu(宋玉), Zhang Min-na (张敏娜), et al. Synthesis of cesium tungsten bronze and its catalytic performance for the dehydration of glycerol to acrolein( 铯铇青铜的合成及其对甘油脱水 制丙烯醛的催化性能) [J]. Chem (化学通报), 2019, 82(11) : 1008-1012.

[55] Drochner A, Ohlig D, Knoche S, et al. Mechanisticstudies on the transition metal oxide catalysed partial oxidation of (Meth) acrolein to the corresponding carboxylic acids[J]. Top Catal, 2016, 59(17/18) : 1518-1532.

[56] Hua Dong-long (花东龙), Zhuang Xiao-yu (庄晓显), Tong Dong-shen (童东绅), et al. Catalytic dehydration and oxidation of glycerin in series to produce acrylic acid (催化甘油脱水氧化连串反应制丙烯酸) [J]. Prog Chem (化学进展), 2016, 28(Z2)：375-390.

[57] Miller J H, Bhan A. Reaction pathways in acrolein oxidation over a mixed-oxide catalyst $[\mathrm{J}]$. ChemCatChem, $2018,10(22)$ : 5242-5255.

[58] Chieregato A, Soriano M D, Basile F, et al. One-pot glycerol oxidehydration to acrylic acid on multifunctional catalysts: Focus on the influence of the reaction parameters in respect to the catalytic performance $[\mathrm{J}]$. Appl Catal B-Environ, 2014, 150/151 : 37-46.

\title{
Research Progress of Composite Metal Oxide Catalysts for the Oxidation of Glycerol to Acrylic Acid
}

\author{
LU Zi-xuan ${ }^{1}$, LI Shuang-ming ${ }^{1,2}$, YU San-san ${ }^{1,2}$
}

(1. College of Chemical Engineering, Shenyang University of Chemical Technology, Shenyang 110142, China; 2. Key Laboratory of Chemical Separation Technology of Liaoning Province, Shenyang 110142, China)

\begin{abstract}
With the shortage of fossil fuels and the environmental problems caused by their utilization, renewable biomass resources have gradually become an important source for the production of fuel and chemicals. In recent years, as the main by-product of biodiesel production, glycerol has attracted widespread attention. The utilization of glycerol to produce high value-added products and the development of relevant conversion technologies have also become a hot spot for scholars at home and abroad. Among many conversion technologies, the selective oxidation of glycerin to acrylic acid has shown broad development prospects. It has important economic and social significance to study the reaction and especially the catalyst. This article summarizes the current research status of composite metal oxide catalysts for the selective oxidation of glycerol to acrylic acid. The current types of catalytic systems used in the reaction and the understanding of the catalytic reaction mechanism of glycerol oxidation to acrylic acid are briefly introduced. Moreover, the existing problems and future prospects are also analyzed and expected.
\end{abstract}

Key words: glycerin; acrylic acid; oxidation reaction; catalyst; reaction mechanism 